\title{
Extended Method of Volume Change Measurements during Solidification of Lamellar Graphite Iron
}

\author{
Péter Svidró ${ }^{1, a^{*}}$, Attila Diószegi ${ }^{1, b}$, Pär G. Jönsson ${ }^{2, c}$ \\ ${ }^{1}$ Department of Materials and Manufacturing, School of Engineering, \\ Jönköping University, Sweden \\ ${ }^{2}$ Department of Material Science and Technology, School of Industrial Engineering and \\ Management, KTH Royal Institute of Technology, Sweden \\ apeter.svidro@ju.se, battila.dioszegi@ju.se, ${ }^{c}$ parj@kth.se
}

Keywords: lamellar graphite iron, volume change, thermal analysis

\begin{abstract}
Lamellar graphite iron (LGI) is an important technical alloy used to produce cast components for the automotive and the marine industry. The performance of the component is defined by the solidification sequence. Therefore, a lot of research work has been done in the field of solidification. The present work introduces a new measurement approach that combines advanced dilatation measurements with thermal analysis to investigate the solidification of LGI. The method involves a thermally balanced spherical sample. The temperature values are measured in the geometrical center and on the surface of the sample. The released heat of solidification is calculated by using the Fourier Thermal Analysis (FTA) method. The displacement values are measured on the surface of the sample. The volume change is calculated from the displacement data. The dilatation results clearly shows the advantage of the multidirectional measurement.
\end{abstract}

\section{Introduction}

The production of a complex shaped LGI casting such as a cylinder head involves the possibility of a casting defect formation such as a shrinkage porosity or a metal expansion penetration. These type of casting defects are related to the volume change during the solidification. The thermal analysis is a basic tool in the investigation of the solidification. Therefore, it is implemented in almost every research application [1-18]. As the sand mold plays an important role in the solidification as the first medium to be in contact with the melt, also several studies have been published on the effect of the mold on the solidification [19-23] together with investigations of the thermo-physical properties of sand mixtures [24]. A long-time known feature that represents the solidification well on macro level is the volume change. Volume change measurement has a longer history than thermal analysis determinations $[25,26]$. Solidification has already been studied with this method, before the application of the ancestor of the modern thermo-electric pyrometer [2729]. All of the volume change methods that can be used for in-situ measurement follow the same principle. Therefore, they may generally be identified as dilatation analysis measurement methods. Here, the movement of the specimen's surface registered and the volume change calculated based on this displacement. This approach usually implements a displacement sensor aligned on the geometrical axis or on the mid-plane of a specimen. Therefore, it is often referenced to as the linear dilatation measurement method. The measurement of the surface movement is usually indirect, where an intermediary medium connects the surface with a displacement sensor. This medium is made of a material with a low linear expansion like sapphire or polycrystalline alumina, to avoid disturbances of the measured results. The linear dilatation measurements are combined with thermal analysis determinations in every experimental setup that is publicly available. Besides certain differences in the design (eg. various layout of the displacement sensors), they follow the same dilatation principle as mentioned above. An extensive literature review and the study of the advantages and drawbacks of the combined thermal and dilatation analysis measurement methods known today leads to the investigation of the limitations of these systems [30]. With a summary of these limitations, different aspects formed as important features that can be improved. 


\section{Classification of the Known Experimental Layouts}

First, the linear dilatation measurements can be classified into two groups based on the applied thermal analysis routine. Most of the experimental setups uses the Newtonian thermal analysis (NTA) method, where the calculations are based on one temperature measurement point. The more advanced setups use the Fourier thermal analysis (FTA) method. It uses at least two cooling curves as the inputs to the calculation $[18,24]$. Consequently, the temperatures are usually registered in the center and near to the surface of the specimen. Several authors have published results that suggests that the FTA method is more reliable than the NTA method, since it is based on a larger number of measured thermal points [14, 31-33].

Second, the linear dilatation measurements can be classified by the number of axes and direction of the displacement recording as it is represented in Figure 1.

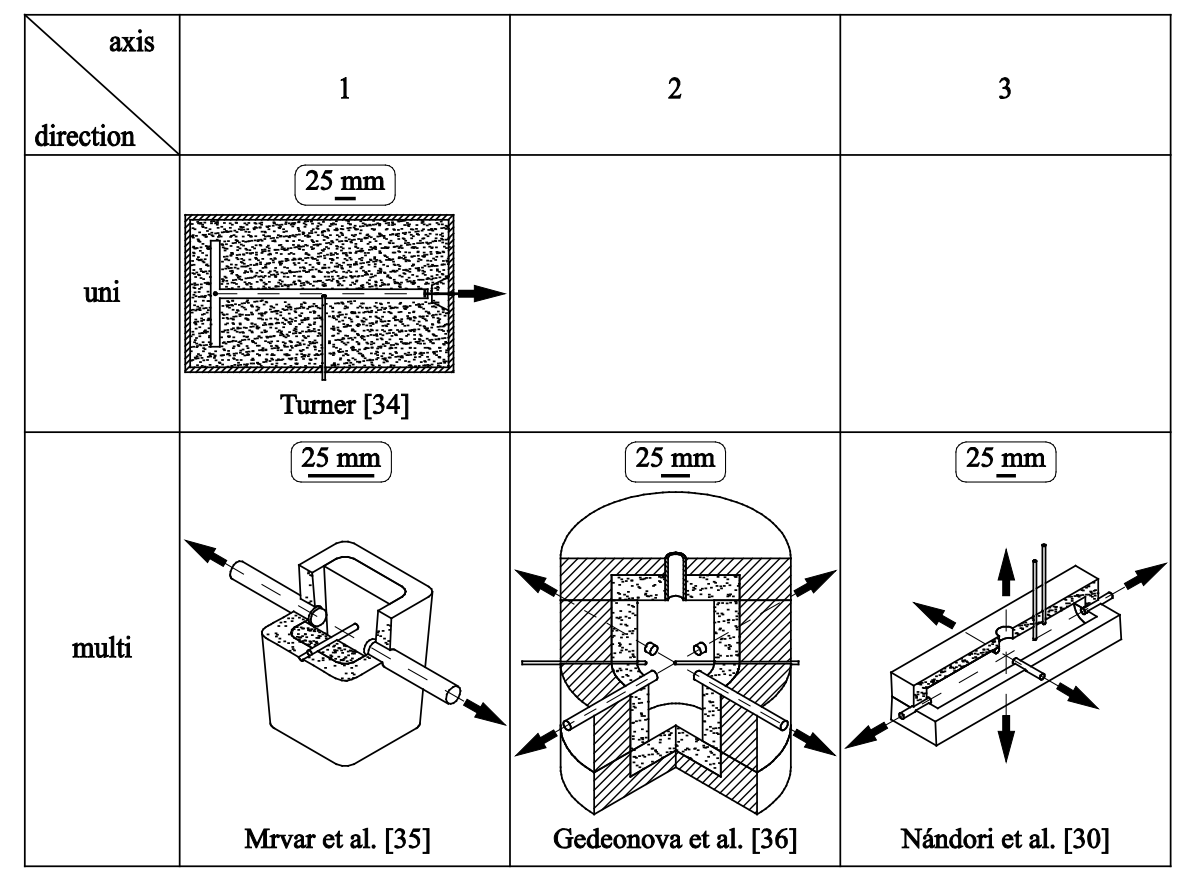

Figure 1. Examples for the classification of linear volume change measurements by the number of axes and the direction of the displacement registration.

\section{Geometry aspects}

The main component in most of the known measurement methods is the specimen holder, which geometric design may vary with the application. The material of the sample holder is usually sand and the investigated alloy is usually cast into a mold. The shape of the sample is a demanding property as it is in strict correlation with the thermal field. Therefore, the design must be symmetric and thermally balanced during the whole solidification period. The shape of the sample or the sample holder must also ensure that the possibility of the right application of the measurement devices.

Thermal aspects

As the solidification proceeds by the heat taken away from the melt, the thermal field of the sample has a huge impact on the solidification. An unbalanced geometry leads to an uneven thermal distribution and this creates a disturbed spot in the thermal field. This spot may move during the solidification [37]. As the thermocouple in the center point is placed in a fixed position, the moving thermal spot will distort the observed temperature values. The thermal field is also heavily correlated to the material of the sample holder, since this is the medium that transports away the heat. As it was mentioned above, in the case of all research units this material is the sand. Several factors influencing the thermal properties of the mold, such as the gas formation of the binder system and the granular nature of the ordinary sand mold. 


\section{Dilatation aspects}

A previous research work on a cylindrical sample shape has shown, that the solidification has an anisotropic nature on a thermally unbalanced specimen [30]. This attribute must be minimized or eliminated by the design of the sample holder. A comparison on the volume change values showed, that the result calculated only with the axial direction $\Delta \mathrm{V}_{\mathrm{A}}$ data is different than calculated with the axial and the radial direction $\Delta_{\mathrm{VA}+\mathrm{R}}$ data, as shown in Figure 2.

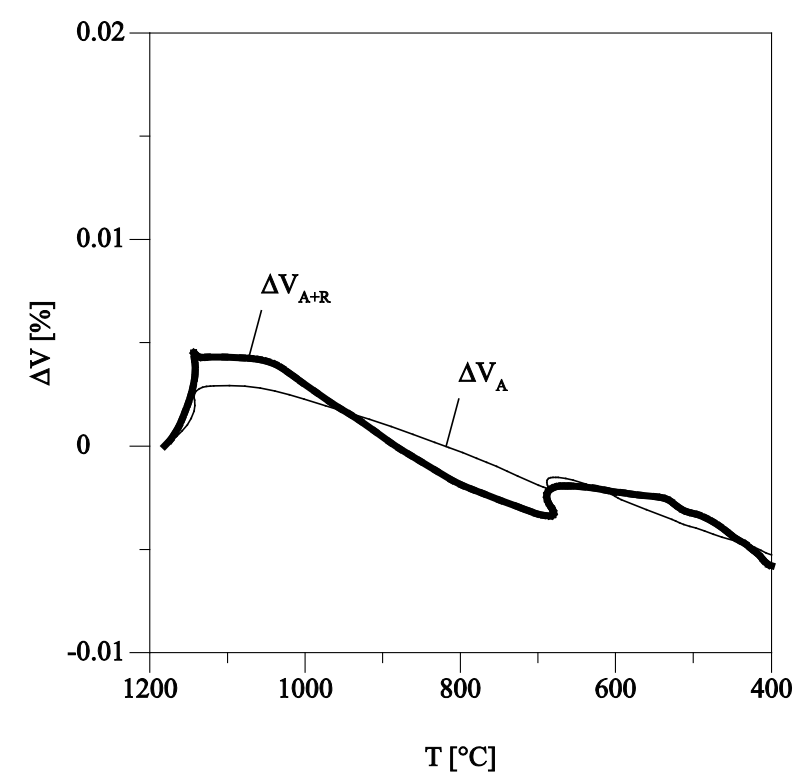

Figure 2. Volume change based on the axial vs. axial and radial displacement values as a function of temperature.

The difference comes from the anisotropy of the solidification, which is a result of the specimen geometry. The radial displacement is a result of the interaction between the internal equiaxed grains, the cylindrical mantle of the columnar zone, and the molding material. The axial displacement is more complicated because in addition to the molding material and the equiaxed grains the axial deformation of the columnar mantle is influenced by the columnar zone forming a disk-shaped zone at both ends of the cylinder. The equiaxed grains are expected to have a random orientation which should have an equally distributed influence on the total radial and axial displacement. Consequently, the measurement layout must consider the right number of measuring directions and devices. Moreover, it must consider the right application of the push rods. It was reported by almost all the authors, that implementation of push rods into the sampler holder (sand mold) wall may provide misleading results at the beginning of the solidification. This is due to the metallostatic pressure and the thermal load of the sand wall.

\section{Experimental Layout}

The summary of the aspects and features above lead to the concept of a spherical design of the specimen. The sampler components and the arrangement of the measuring sensors are presented in Figure 3. 


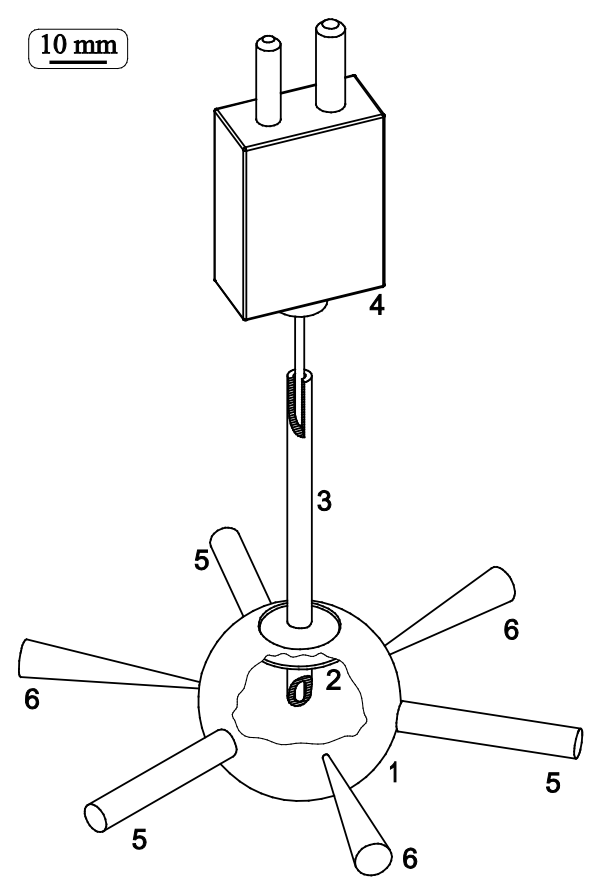

Figure 3. Schematic of the sampler geometry and the arrangement of the sensors.

1 - Spherical liquid collector made of steel with a wall thickness of $0.5 \mathrm{~mm}$.

2 - Lid made of the same material and the same wall thickness as the spherical liquid collector. The diameter of the lid is $18 \mathrm{~mm}$ and it is welded to a steel tube.

3 - Steel tube made of the same material as the spherical liquid collector, which is closed at one end. The outer diameter of the tube is $4 \mathrm{~mm}$ and the wall thickness is $1 \mathrm{~mm}$.

4 - Mineral Insulated N-type thermocouple. The diameter of the thermocouple wire is $1.5 \mathrm{~mm}$.

5 - Push rod made of engineering grade full-fired $99.8 \%$ polycrystalline alumina. Three push rods transmit the displacement of the surface to the linear variable differential transformer (LVDT). The LVDT has a measuring range of $+-3 \mathrm{~mm}$, a sensitivity of $85.20 \mathrm{mV} / \mathrm{V} \mathrm{mm}$, and a linearity of $+-0.05 \%$ for a full scale output.

6 - Infrared (IR) thermometer (3x). The IR unit is equipped with a two-color spectral sensor and has a measuring range of $500-1400^{\circ} \mathrm{C}$.

In Figure 3 the sampler is closed by the gravity as the open sphere lays on the lid. The sample holder is used as an immersion sample collector. The sampling starts with the immersion of the sampler into the melt. At that point, the melt can enter the cavity through the top opening. As soon as the hollow body is filled, it can be removed from the melt. By the raise of the sampler, the lid will lock the sample as a closed volume. Temperature measurements as well as displacement units can be applied on the surface as soon as the sampler is out from the melt. The geometric mid-plane of the sphere equals to the inner surface of the tube where the central thermocouple is placed. As the lid has the same curvature of the sphere, the thermocouple will always be in the thermal center no matter how the sphere turns. Also, the thermocouple can be released after the completion of the measurements. The spherical sampler design also makes possible to apply thermocouples and push rods on the surface without embedment.

The melt was poured from a $2 \mathrm{~kg}$ capacity pre-heated hand cup. The temperature of the melt was $1420{ }^{\circ} \mathrm{C}$. A spherical sample was submerged into a melt in a hand-held cup for 2.5 seconds. Thereafter, the measurement was started as the push rods touched the surface of the sampler. The signals from the thermocouple, the IRs and the LVDTs were collected by a unique controller system with sampling rate of ten records per seconds. The experiment involves one alloy, which chemical composition is given in Table 1 .

\begin{tabular}{ccccccccc}
\hline Element & $\mathrm{C}$ & $\mathrm{Si}$ & $\mathrm{Mn}$ & $\mathrm{P}$ & $\mathrm{S}$ & $\mathrm{Cr}$ & $\mathrm{Cu}$ & $\mathrm{CE}$ \\
\hline $\mathrm{wt} \%$ & 3.20 & 1.88 & 0.61 & 0.03 & 0.03 & 0.15 & 0.28 & 3.85 \\
\hline
\end{tabular}


Figure 4 shows the cross section of the sampler which was split by a disc cutter.

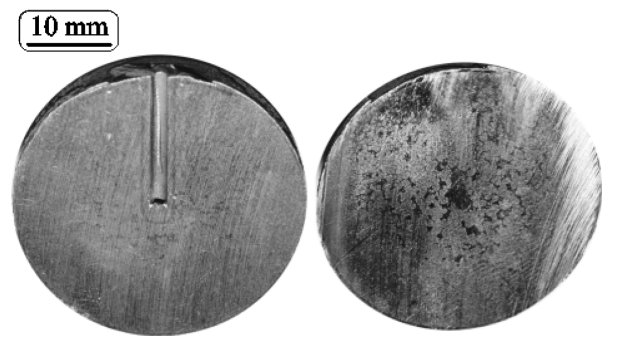

Figure 4. Raw cut surfaces of the sample.

\section{Results and Discussion}

The displacement values collected in the solidification experiment are presented in Figure 5.

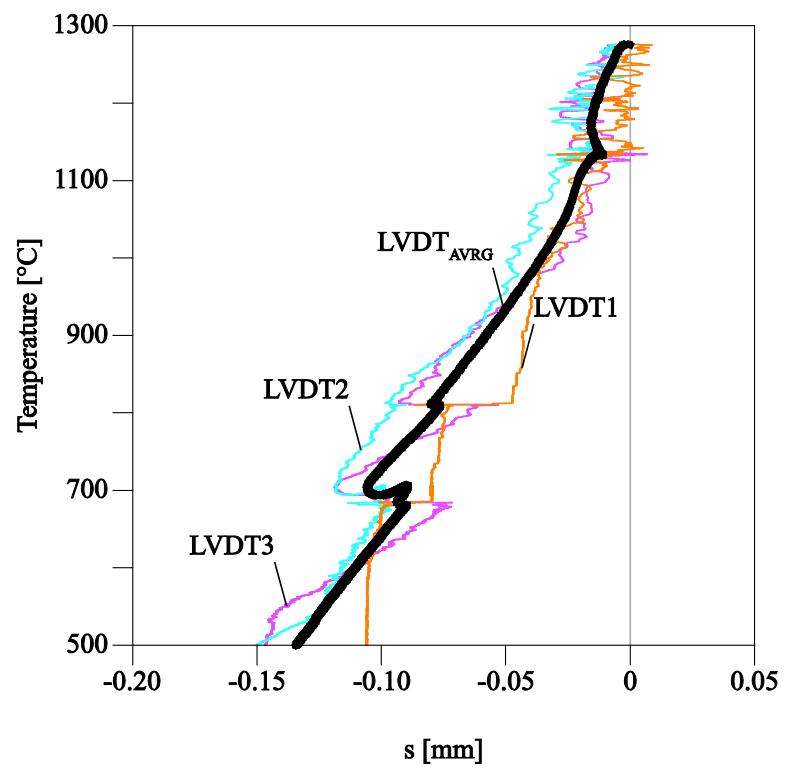

Figure 5. Displacement values as a function of temperature.

It is important to highlight the importance of the multiaxial and multidirectional displacement measurements. The data from the individual LVDTs shows altering behavior of the surface of the sample. These displacement curves looks like a data with heavy noise and contradictory movements. However, due to the thermally balanced specimen, the movements are counteracting each other. Therefore, the average of the data from the displacement sensors shows a smooth displacement curve without any additional signal conditioning. The temperature values collected in the experiments are presented in Figure 6. 


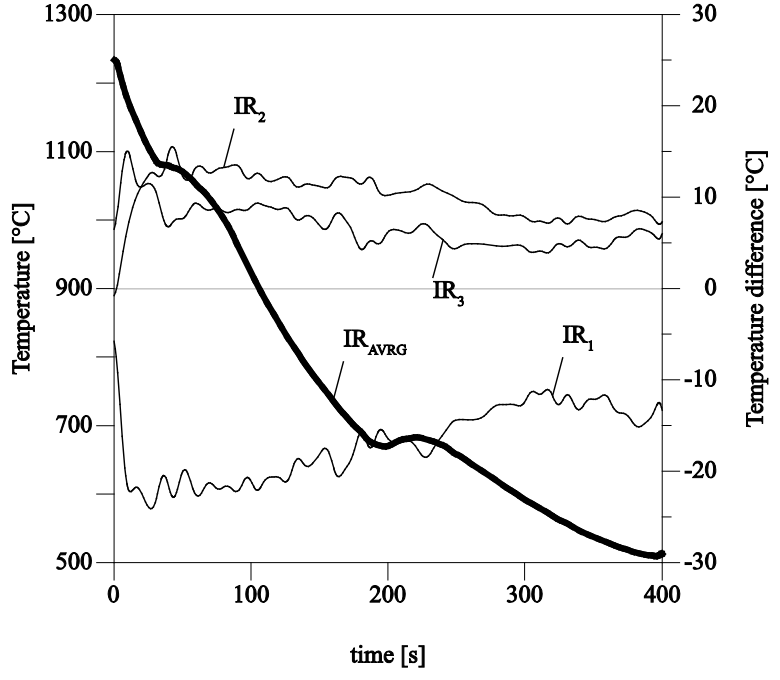

Figure 6. Temperature values as a function of time.

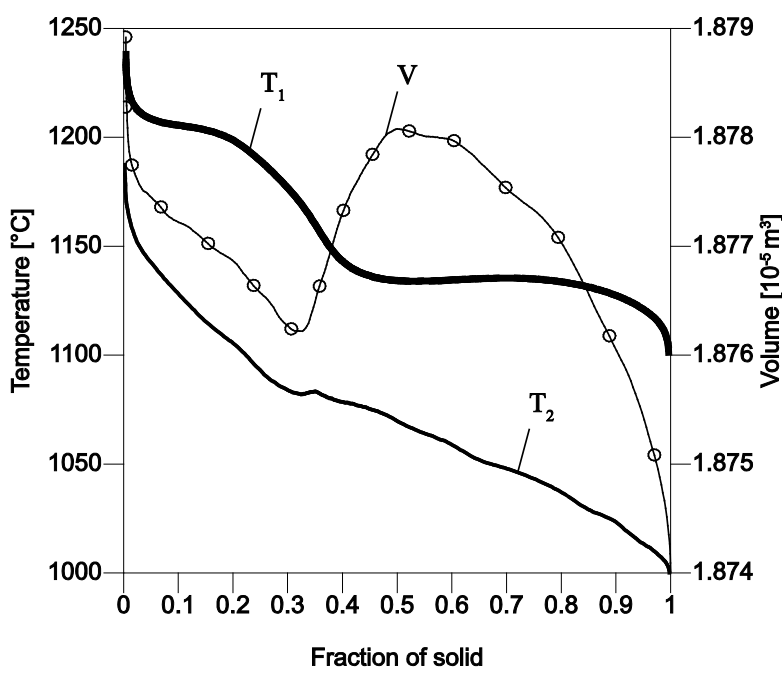

Figure 7. Cooling curves and the calculated volume at different solid fractions in the melt.

The temperature values from the IR sensors shows an even heat distribution on the surface of the sample. In Figure 6, $\mathrm{IR}_{\mathrm{AVRG}}$ is the average temperature value calculated from three IR sensors. The $I_{1}, I R_{2}$, and $I_{3}$ curves represent the temperature difference of the individual IR temperature measurement spots from the average value. In Figure 7, the cooling curves and the calculated sample volume variation based on the measured displacements are plotted as a function of the fraction solid calculated by FTA. The thermal properties used in the calculations are $c_{\mathrm{p}}^{\text {liq }}=740 \mathrm{Jkg}^{-1} \mathrm{~K}^{-1}, \rho^{\text {liq }}=7100 \mathrm{kgm}^{-3}, c_{\mathrm{p}}^{\text {aus }}=710 \mathrm{Jkg}^{-1} \mathrm{~K}^{-1}, \rho^{\text {aus }}=7400 \mathrm{kgm}^{-3}, c_{\mathrm{p}}^{\text {gr }}=1950 \mathrm{Jkg}^{-1} \mathrm{~K}^{-1}$, $\rho^{\mathrm{gr}}=2200 \mathrm{kgm}^{-3}, c_{\mathrm{p}}$ calculated by ThermoCalc software [38], $\rho$ according to [39].

The parameter $\mathrm{T}_{1}$ denotes the cooling curve registered from the geometrical centrum of the spherical sample, $T_{2}$ denotes the average of the temperatures measured on the surface of the sample. The contraction from the beginning of the solidification to $f_{s}=0.32$ occurs as the columnar zone (casting skin) is formed on the internal surface of the casting sampler as it solidifies. At $\mathrm{f}_{\mathrm{s}}=0.32$ the $\mathrm{T}_{2}$ value indicates a temperature minimum. This value is known as the beginning of the eutectic phase transformation in the columnar zone. In the intradendritic area of the columnar zone the graphite precipitates and cause the expansion of the whole sample. The consequent volume increase continues until the internal domain (the equiaxed zone) reaches a temperature minimum on the central cooling curve $T_{1}$ at $f_{s}=0.52$. This value represents the beginning of eutectic precipitation in this domain. From this moment, the total volume of the sample will decrease towards to the end of the solidification.

\section{Conclusions}

A novel measurement method was introduced for volume change measurements during solidification of LGI. The new setup considers the anisotropic property of the solidification and it eliminates the effect of the mold material on the solidification. The importance of the multiaxis measurement was highlighted, together with the symmetrical distribution of the displacement sensors. It was shown, that the spherical geometry is thermally balanced. Therefore, the specimen excludes the error from the measurement that a migrating hotspot can create.

\section{Acknowledgement}

The present work was performed within the Casting Innovation Centre and it was financed by the Swedish Knowledge Foundation. Cooperating parties in the project are Jönköping University, Scania CV AB and Volvo Group Trucks Operations. The participating persons from these institutions/companies are acknowledged for their support throughout this study. 


\section{References}

[1] T. F. Russell, The interpretation of thermal curves and some applications to ferrous alloys, J. Iron Steel Inst. 139 (1939) 147-186.

[2] B. J. Hribovsek, B. Marincek, Thermal analysis of bulk solidified cast iron melts, International Symposium on the Metallurgy of Cast Iron, St Saphorin, Switzerland, 1975, pp. 659-672.

[3] L. Bäckerud, K. Nilsson, H. Steen, Study of Nucleation and Growth of Graphite in Magnesium Treated Cast Iron by Means of Thermal Analysis, International Symposium on the Metallurgy of Cast Iron, St Saphorin, Switzerland, 1975, pp. 625-637.

[4] H. Fredriksson, B. Rogberg, Thermal analysis for interpretation of solidification cycle, Met. Sci. 13 (12) (1979) 685-690.

[5] U. Ekpoom, R. Heine, Thermal Analysis by Differential Heat Analysis (DHA) of Cast Iron, Am. Foundrymens Soc. 1981 AFS Res. Rep., pp. 49-60, 1982.

[6] K. G. Upadhya, D. M. Stefanescu, K. Lieu, D. P. Yeager, Computer-aided Cooling Curve Analysis, Principles and Applications in Metal Casting, AFS Transactions 97 (1989) 61-66.

[7] Fred J. Bradley, Conrad A. Fung, Thermal Analysis for shrinkage prediction in commercial ductile iron castings, Can. Metall. Q. 30 (4) (1991) 251-260.

[8] E. Fras, W. Kapturkiewicz, H. F. Lopez, The solidification kinetics of cast iron using an improved thermal analysis technique, Int. J. Cast Metal. Res. 6 (3) (1993) 137-142.

[9] L. Battezzati, M. Baricco, F. Marongiu, G. Serramoglia, D. Bergesio, Melting and Solidification Studies by Advanced Thermal Analysis of Cast Iron, Metall. Sci. Technol. 19 (2) (2001) 16-20.

[10] A. Diószegi, J. Hattel, Inverse thermal analysis method to study solidification in cast iron, Int. J. Cast Metal. Res. 17 (5) (2004) 311-318.

[11] E. Fraś, M. Górny, H. F. López, The transition from gray to white cast iron during solidification: Part III. Thermal analysis, Metall. Mater. Trans. A 36 (11) (2005) 3093-3101.

[12] D. Emadi, L. V. Whiting, S. Nafisi, R. Ghomashchi, Applications of thermal analysis in quality control of solidification processes, J. Therm. Anal. Calorim. 81 (1) (2005) 235-242.

[13] A. Diószegi, I. L. Svensson, Interpretation of Solidification by Thermal Analysis of Cooling Rate, Trans. Indian Inst. Met. 58 (4) (2005) $611-616$.

[14] A. Diószegi, I. L. Svensson, On the problems of thermal analysis of solidification, Mat. Sci. Eng. A - Struct 413-414 (2005) 474-479.

[15] P. Larrañaga, J. M. Gutiérrez, A. Loizaga, J. Sertucha, R. Suárez, A Computer-Aided System for Melt Quality and Shrinkage Propensity Evaluation Based on the Solidification Process of Ductile Iron, AFS Transactions 116 (2008) 547-561.

[16] I. Riposan, M. Chisamera, S. Stan, M. Barstow, Identifying chill tendency of cast iron melts by thermal analysis, Int. J. Cast Metal. Res. 26 (3) (2013) 152-159.

[17] J. F. Xu, F. Liu, D. Zhang, Z. Y. Jian, An analytical model for solidification of undercooled metallic melts, J. Therm. Anal. Calorim. 119 (1) (2015) 273-280.

[18] A. Diószegi, V.-L. Diaconu, V. Fourlakidis, Prediction of volume fraction of primary austenite at solidification of lamellar graphite cast iron using thermal analyses, J. Therm. Anal. Calorim. 124 (1) (2016) 215-225.

[19] S. Engler, D. Boenisch, B. Kohler, Metal and Mold Wall Movements During Solidification of Cast Iron, Cast Metals Research Journal AFS 9 (1973) 20-30.

[20] M. Chisamera, I. Riposan, S. Stan, P. Toboc, T. Skaland, D. White, Shrinkage evaluation in ductile iron as influenced by mould media and inoculant type, Int. J. Cast Metal. Res., 24 (1) (2011) 28-36.

[21] Y. Zou, Y. Hayashi, H. Kojima, K. Yamazaki, H. Nakae, Influence of Mold Material and CE Value on Shrinkage of Spheroidal Graphite Cast Iron, presented at the 70th World Foundry Congress, Monterrey, Nuevo Leon, Mexico, 2012.

[22] S. Stan, M. Chisamera, I. Riposan, M. Barstow, Application of thermal analysis to monitor the quality of hypoeutectic cast irons during solidification in sand and metal moulds, J. Therm. Anal. Calorim. 110 (3) (2012) 1185-1192. 
[23] G. Alonso, D. M. Stefanescu, R. Suarez, A. Loizaga, G. Zarrabeitia, Understanding graphite expansion during the eutectic solidification of cast iron through combined Linear Displacement and Thermal Analysis, Giessereiforschung 66 (2014) 18-29.

[24] J. T. Svidró, A. Diószegi, J. Tóth, The novel application of Fourier thermal analysis in foundry technologies, J. Therm. Anal. Calorim. 115 (1) (2014) 331-338.

[25] R.-A. F. de Réaumur, Memoirs on steel and iron. 1722.

[26] R. Mallet, On the Alleged Expansion in Volume of Various Substances in Passing by Refrigeration from the State of Liquid Fusion to That of Solidification, P. R. Soc. London, 23 (1874) 209-234.

[27] Thomas Johann Seebeck, Abhandlurgen Phys. Kl. Koniglichen Akad. Wiss. Zu Berl., vol. 1822-23, pp. 265-373.

[28] Henry Le Chatelier, Comptes Rendus, 102 (1886) 819-822.

[29] Henry Le Chatelier, J. Phys, vol. 6, p. 23, 1887.

[30] P. Svidró, A. Diószegi, On problems of volume change measurements in lamellar cast iron," Int. J. Cast Metal. Res. 27 (1) (2013) 26-37.

[31] E. Fras, W. Kapturckiewicz, A. Burbielko, H. F. López, Numerical simulation and fourier thermal analysis of solidification kinetics in high-carbon Fe-C alloys, Metall. Mater. Trans. B 28 (1) (1997) 115-123.

[32] A. Diószegi, I. L. Svensson, A comparison of Fourier vs. Newtonian thermal analyse and its influence on the inverse kinetic growth calculation, in A. Diószegi: Dissertation No. 871, Linköping, Sweden, 2004, pp. 311-318.

[33] D. Emadi, L. V. Whiting, M. Djurdjevic, W. T. Kierkus, J. Sokolowski, Comparison Of Newtonian And Fourier Thermal Analysis Techniques For Calculation Of Latent Heat And Solid Fraction Of Aluminum Alloys, Metal. - J. Metall. 2 (10) (2004) 91-106.

[34] T. Turner, Volume and Temperature Changes during the Cooling of Cast Iron, J. Iron Steel I. 1 (1906) 48-74.

[35] P. Mrvar, M. Trbižan, J. Medved, Investigation of cast iron solidification with dilatation analysis, Kovine Zlitine Tehnol. Slov. 33 (1-2) (1999) 45-49.

[36] Z. Gedeonova, S. Bodi, J. Dúl, G. Nándori, L. Vigh, Displacement on the surface mould and metal during the solidification of nodular graphite iron castings, Mater. Sci. Forum 2 (215216) (1995) 391-398.

[37] L. Elmquist, A. Diószegi, On the Problems of a Migrating Hot Spot, Mater. Sci. Forum, 649 (2010) 443-448.

[38] Thermo-Calc 2016b, database: TCFE7 - Steels/Ferrous Alloys. Stockholm, Sweden: ThermoCalc AB.

[39] I. L. Svensson, I. Dugic, Modelling of volumes in cast iron solidification to predict shrinkage and expansion defects, Int. J. Cast Metal. Res. 11 (6) (1999) 489-494. 